\title{
The problem of the acceleration of electrons of the outer radiation belt and magnetospheric substorms
}

\author{
E. E. Antonova ${ }^{1 *}$ and M. V. Stepanova ${ }^{2}$
}

\begin{abstract}
Predicting of the location of the maximum in high-energy electron fluxes filling a new radiation belt is an endeavor being carried out by physicists studying the magnetosphere. We analyzed the data from the Defense Meteorological Satellite Program (DMSP) satellites and ground-based magnetometers obtained during geomagnetic storm on 8-9 October 2012. The minimum value of the disturbance storm time (Dst) was -111 nT, and the maximum in high-energy electron fluxes that appeared during the recovery phase was observed at $L=4 \operatorname{Re}$. At the same time, we analyzed the motion of the auroral oval toward lower latitudes and related substorm activity using the data of the low-orbiting DMSP satellites and the IMAGE magnetic meridian network. It was found from the DMSP satellites' measurements that the maximum of the energy density of precipitating ions, the maximum of the plasma pressure, and the most equatorial part of the westward auroral electrojet are all located at the $60^{\circ}$ geomagnetic latitude. This value corresponds to $L=4$ Re, i.e., it coincides with the location of the maximum in high-energy electron fluxes. This $L$-value also agrees with the predictions of the Tverskaya relation between the minimum in Dst variation and the location of the maximum of the energetic electron fluxes, filling a new radiation belt. The obtained results show that the location of this maximum could be predicted solely from the data of the auroral particle precipitations and/or ground-based magnetic observations.
\end{abstract}

Keywords: Magnetospheric storm and substorm; Outer radiation belt; Auroral oval

\section{Introduction}

Large fluxes of relativistic electrons in the magnetosphere of the Earth fill the outer radiation belt (ORB). Their properties were studied using the data of many satellites including the recently launched Van Allen (Radiation Belt Storm Probes (RBSP)) mission. However, the problem of the formation of the ORB remains relevant. It is well known (see, for example, Reeves (1998)) that large fluxes of relativistic electrons in the ORB appear during the recovery phase of a geomagnetic storm. Nevertheless, this phenomenon does not always take place. Reeves et al. (2003) showed that geomagnetic storms can either increase or decrease the fluxes of relativistic electrons in the radiation belt. They stressed that the net effect of geomagnetic storms on

\footnotetext{
* Correspondence: antonova@orearm.msk.ru

${ }^{1}$ Skobeltsyn Institute of Nuclear Physics, Lomonosov Moscow State University, Moscow, Russia

Full list of author information is available at the end of the article
}

radiation belt fluxes is a delicate and complicated balance between the processes leading to particle acceleration and loss.

Development of geomagnetic storms is accompanied by an increase of pressure inside the magnetosphere and ring current formation. During storms, intense substorms are usually observed. Magnetic field line dipolarizations during those substorms create seed populations of electrons (see, Baker et al. 2005, Kissinger et al. 2014, and references therein). Later, they are accelerated, and a new ORB is formed. This means that the dynamics of the ORB is strongly affected by the storm-time substorms. All substorms (during storms and in the absence of storms) develop in a region that is mapped into the auroral oval. Therefore, the analysis of the motion of the auroral oval during storms could provide very useful information on the storm/substorm dynamics. Since the report by Akasofu and Chapman (1963), it is well known that during magnetic storms the auroral oval moves 
toward lower latitudes. Newell et al. (2009) showed that when the geomagnetic activity increases, the form of the auroral oval becomes more circular. This feature of the auroral oval is difficult to understand within the traditional view of mapping of the auroral oval into the plasma sheet proper. This was resolved by Antonova et al. $(2013,2014 a, b)$ who have recently shown that a major part of the oval is mapped into a plasma ring which contains transverse currents surrounding the Earth. In this approach, the circular form of the oval is natural. This finding could be relevant for understanding the storm time auroral dynamics.

Reeves et al. (2013) studied the evolution of phase space density of relativistic electrons during 8-9 October 2012 geomagnetic storm and clearly demonstrated that the region of acceleration of the ORB electrons is located in the inner magnetosphere. They attributed the acceleration of the ORB electrons to the VLF whistler-mode chorus waves. In fact, intense wave activity was observed during this storm. Reeves et al. (2013) carried out a very careful analysis of the evolution of the phase space density of relativistic electrons but did not study the auroral dynamics during this event. However, as shown by Reeves et al. (2003), for many storms, the net effect of storm on electron acceleration is very low (see Figure $2 \mathrm{c}$ in Reeves et al. 2003). The electron fluxes decrease during the main phases of such storms and regain pre-storm levels when a storm ends. This means that large-scale variations in the magnetic field during the storm are very important for the processes of particle acceleration and deceleration.

Magnetic field variations at the equatorial plane cannot be adequately studied using the data of the Van Allen probe mission in most cases due to orbit inclination, and Reeves et al. (2013) used the TS04 model (Tsyganenko and Sitnov, 2005) for the determination of the phase space density of relativistic electrons. Fluxes of low energy particles were not analyzed. However, it would be interesting to make this analysis in the future because increases of low-energy fluxes lead to the depression of the magnetic field during the ring current development. Restore of the field during the ring current decay will lead to particle acceleration. Antonova et al. (2014a,b) argued that most part of the auroral oval is mapped into the high latitude part of the ring current. That is why it is interesting to analyze the position of the auroral oval for the Reeves et al. (2013) storm.

In this paper, we analyze the position of the auroral oval and the westward auroral electrojet for the 8-9 October 2012 magnetic storm, using the data of the Defense Meteorological Satellite Program (DMSP) satellites and the International Monitor Geomagnetic Effects (IMAGE) magnetometer network, and show that the analysis of such observations can be very important with regard to solving of the problem of the acceleration of electrons in the ORB.

\section{The 8-9 October 2012 magnetic storm and the position} of the auroral oval and of the westward auroral electrojet Figure 1a shows the solar wind velocity, density, dynamic pressure, and three components of the interplanetary magnetic field (IMF), obtained using the 5-min resolution data from the OMNIweb interface (King and Papitashvili 2005). The 8-9 October 2012 strong geomagnetic storm was caused by two comparatively long periods of the southward IMF orientation: between October 7 16:32 UT and October 8 12:56 UT and between October 8 18:36 UT and October 9 10:02 UT, during which there occurred a strong decrease in the values of the disturbance storm time (Dst) index. Reeves et al. (2013) showed that before the main phase of the storm the fluxes of relativistic electrons in ORB were very low. This decrease is attributed to a previous storm that took place between 30 September and 1 October 2012. Starting from that time, the electron fluxes remained very low. They continued to decrease very slowly until early morning on 9 October following when they began to increase. It is necessary to mention that on 7 October between 3:00 and 18:45 UT, the solar wind density and dynamic pressure were comparatively high, having maximum values $26 \mathrm{~cm}^{-3}$ and $7.2 \mathrm{nPa}$, respectively (see Fig. 1a). However, the value of Dst variation corrected by the solar wind dynamic pressure (red line in Fig. 1b) Dst $^{*}=$ Dst $-b\left(P_{d}\right)^{1 / 2}+c$ (Burton et al. 1975) does not differ significantly from the ordinal Dst variation (blue line in Fig. 1b), and hence the variation in Dst index is mainly related to ring current dynamics. Figure 1c shows the Dst and SYM-H, AL, and AU geomagnetic indexes, obtained from the OMNI 5-min resolution database (http://omniweb.gsfc.nasa.gov/ow_min.html), and the PC index, obtained from the Arctic and Antarctic Research Institute and Technological University of Denmark (http://pc-index.org/). Storm-time substorms were observed when the IMF $\mathrm{Bz}$ had a southward orientation resulting in the injection of the seed population of electrons. Some periods of decrease in the module of SYM-H could be attributed to the changes in the sign of IMF Bz. The true recovery phase began on 9 October at 8:28 UT when the Bz component started turning northward.

Reeves et al. (2013) showed that the period of ORB acceleration had a duration of approximately $11 \mathrm{~h}$ (from 23:17 UT on 8 October till 13:02 UT on 9 October) and that the center of the region of acceleration was located at $L^{*}=4$ (where $L^{*}=2 \pi M / \Phi R e, M$ is the Earth's magnetic moment, $R e$ is the radius of the Earth, and $\Phi$ the total magnetic flux enclosed within a drift shell of an electron), where $L^{*}$ does not different greatly from the 
(a)
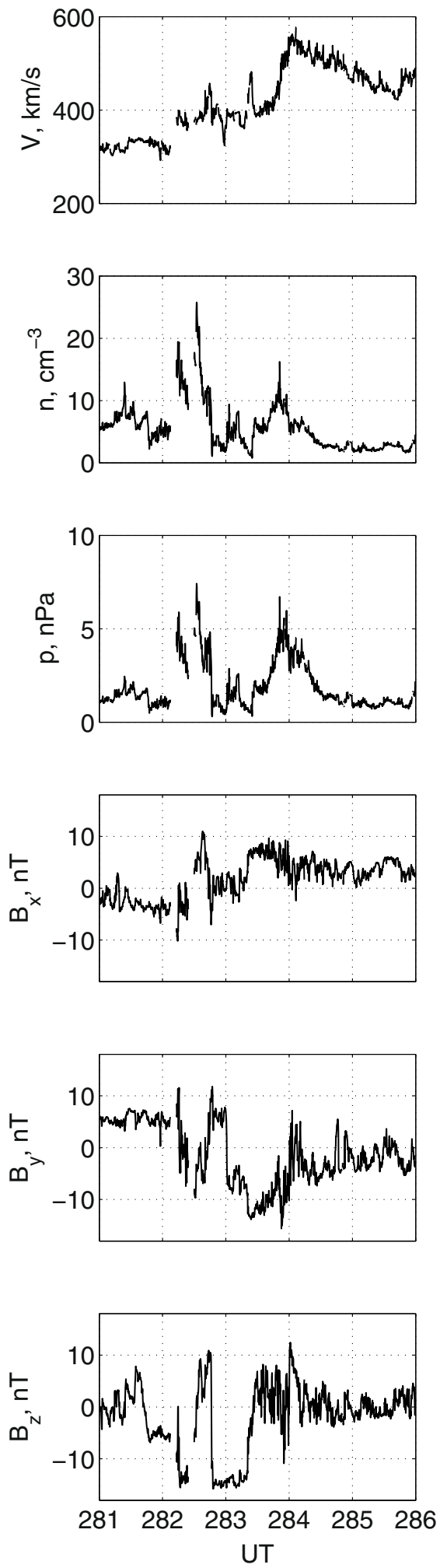

(b)

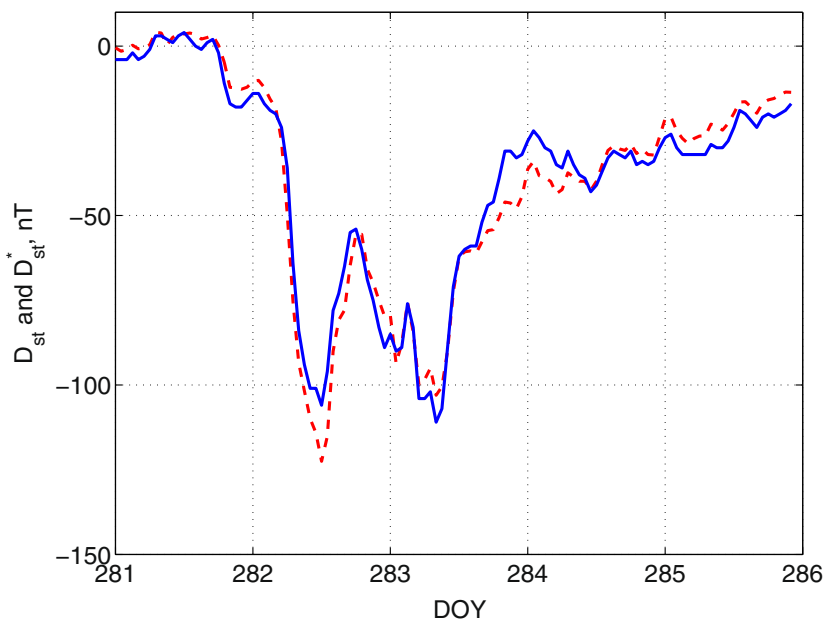

(c)
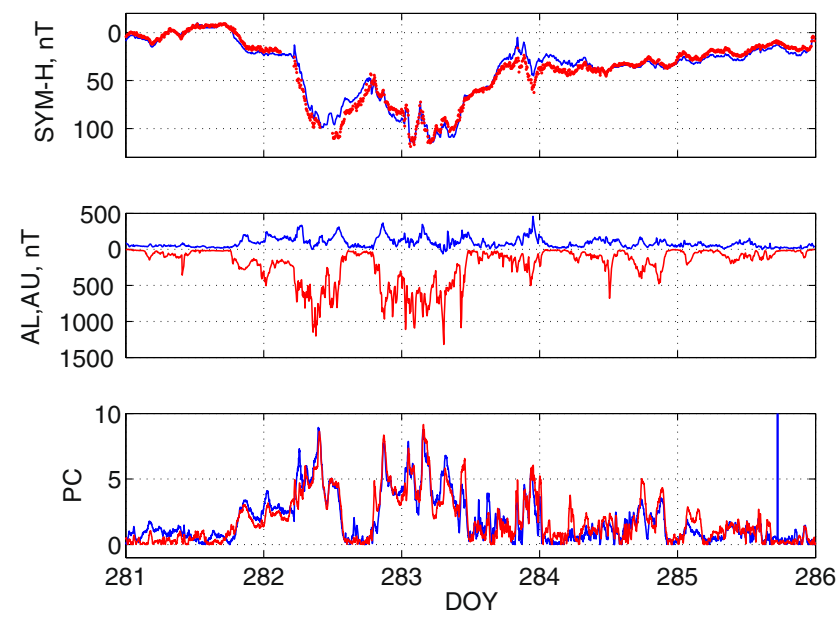

Fig. 1 (See legend on next page.) 
(See figure on previous page.)

Fig. 1 Solar wind parameters and geomagnetic indexes for the geomagnetic storm on 8-9 October 2012. From top to bottom: the solar wind velocity, number density, dynamic pressure, and three components of the interplanetary magnetic field (a), the Dst (blue curve) and Dst* (red curve) corrected by dynamic pressure Dst indexes (b); from top to bottom: the Dst (blue curve) and SYM-H (red curve), AL (red curve), AU (blue curve), and PC (PC north-blue curve, PC south-red curve) indexes (c)

distance to the top of magnetic field line in the equator $(L)$ when the magnetospheric field is nearly dipolar.

To identify the location of the region of acceleration relative to the auroral oval, we analyze the data of auroral precipitations from the DMSP F16, 17, and 18 satellites during the 8-9 October 2012 storm and observe the increase in the thickness of the auroral oval and the shift of its equatorial boundary toward the equator. Figure 2 shows ion and electron energy precipitating fluxes measured by the DMSP F17 satellite during the auroral oval crossing in the night-side southern hemisphere on 8 October 2012. The time of crossing (21:47:40-21:52:50 UT) corresponds to the start of the ORB acceleration in accordance with Reeves et al. (2013, Fig. 2). It is possible to see that the maximum of the precipitating ion energy flux (b2i boundary introduced by Newell et al. (1996)) is located near the $60^{\circ}$ geomagnetic latitude which corresponds to $L=4$, where $L$ is the Mcllwain parameter).

Expansion of the auroral oval toward the equator coincides with the expansion of the auroral electrojet. Figure 3 shows the position of the auroral electrojet using the $1 \mathrm{D}$ equivalent currents obtained from the data of the IMAGE magnetometer network (http://space.fmi.fi/ image/beta/?page=home). It is possible to see that for the same time interval near midnight, the westward auroral electrojet was located at the latitude near the $62^{\circ}$ geographic latitude, i.e., near the $60^{\circ}$ geomagnetic latitude.

The observed coincidence of the maximum of the precipitating ion energy flux and the westward auroral

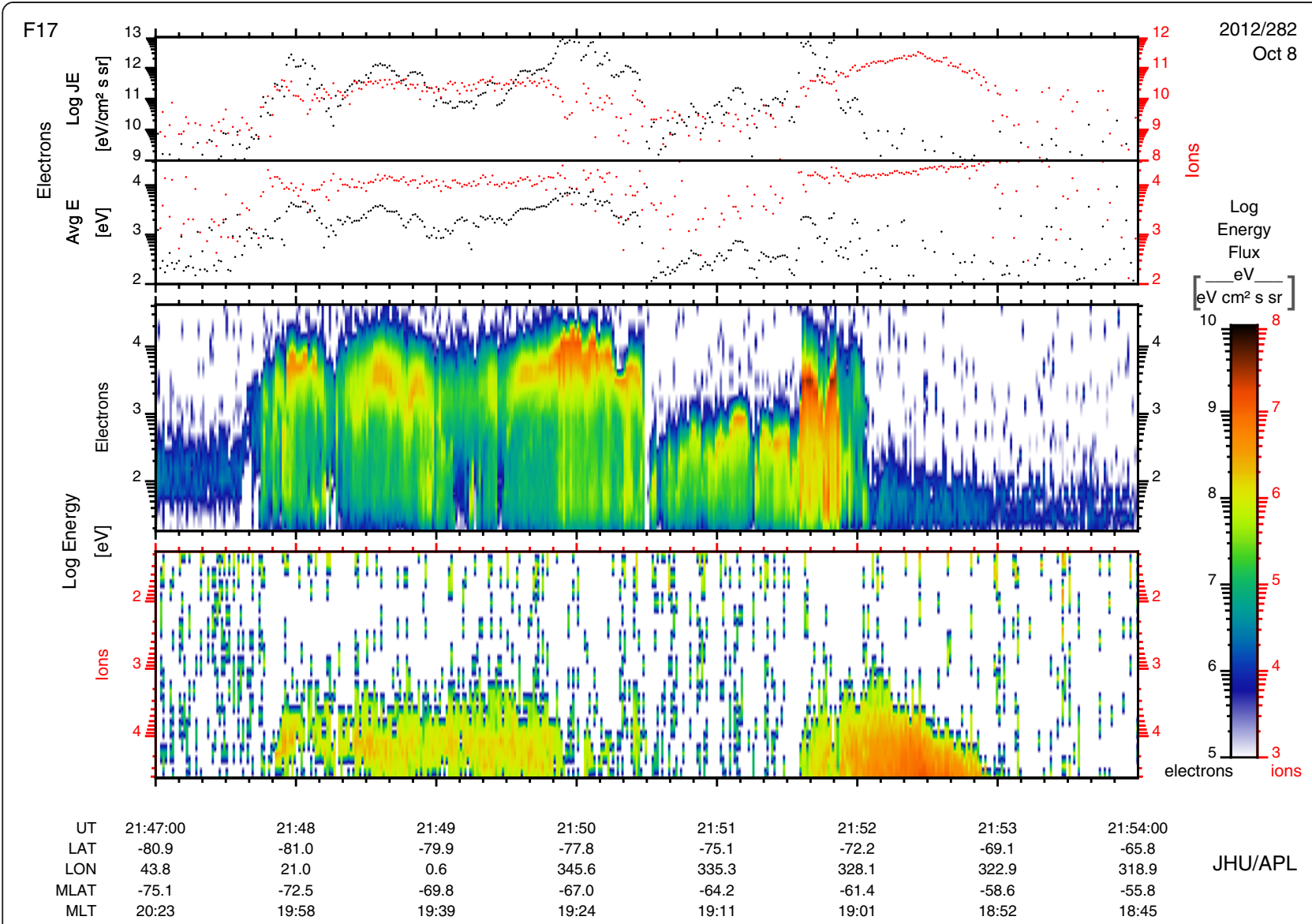

Fig. 2 Electron and ion energy auroral precipitating fluxes measured during the time interval near the Dst minimum. From top to bottom: the electron and ion average energies, electron, and ion precipitating energy fluxes measured by the DMSP F17 satellite on 8 October 2012 during the auroral oval crossing in the Southern Hemisphere between 19 and 20 MLT. The time of crossing is close to the time of one of the minimums of Dst variation 


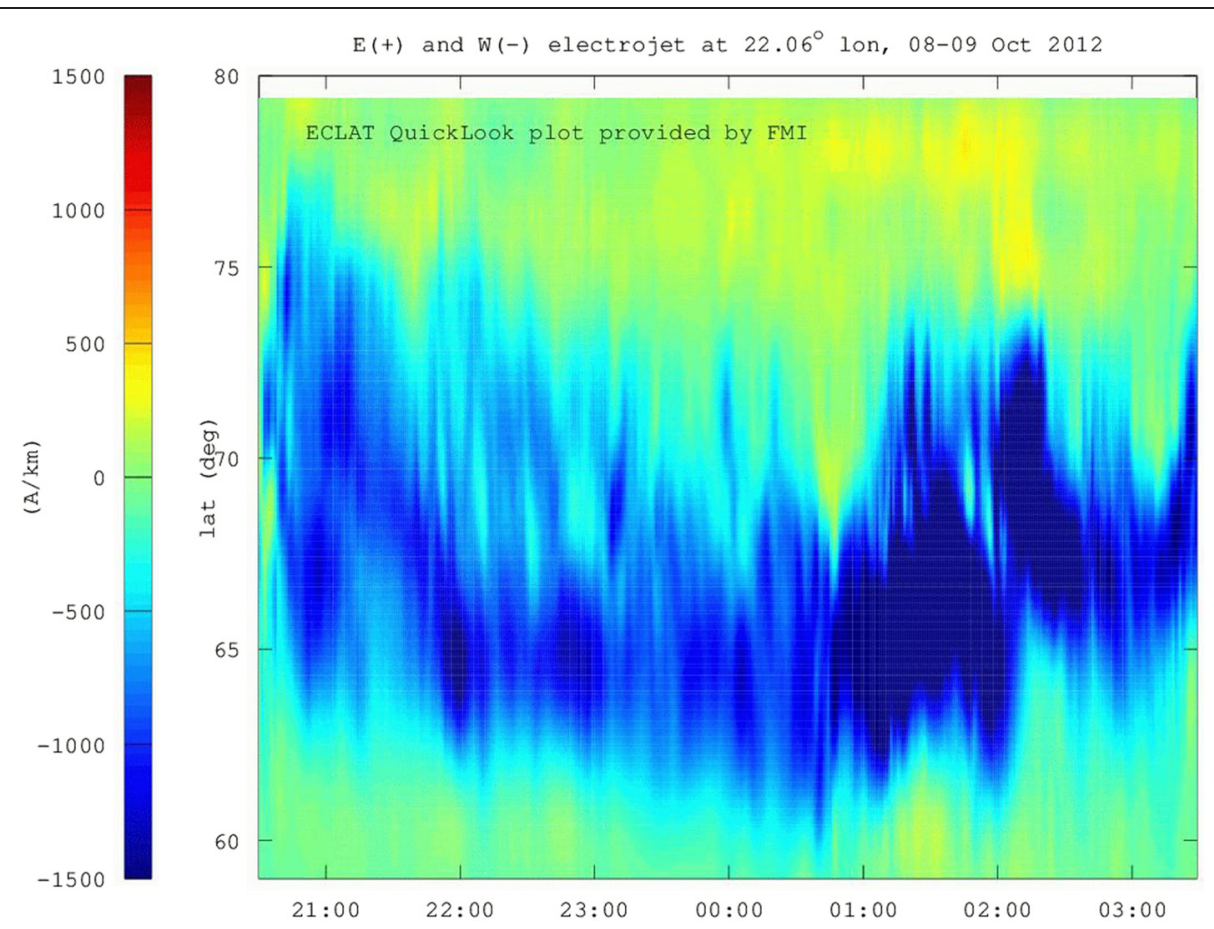

Fig. 3 Intensity of the westward auroral electrojet, obtained using the IMAGE magnetometer network near the Dst minimum. The 1D equivalent current system obtained using the data of the IMAGE magnetometer network on 8-9 October 2012

electrojet is a natural consequence of the processes leading to ring current formation during magnetic storms. The shift of the auroral oval toward lower latitudes leads to the shift of its equatorial boundary and hence of the equatorial boundary of the westward electrojet. At the same time, due to dipolarizations related to the substorm activity, both ions and electrons are accelerated, leading to an increase in particle fluxes. Hence, the observed increase in ion fluxes and the ion pressure occurs in the same place where the seed population of electrons emerges. The seed electrons later get accelerated to relativistic energies. That is why in spite of intense radial diffusion, the $L$-position of the maximum of phase space density of relativistic electrons coincides with the position of the maximum of the precipitating ion energy flux (ion pressure) and the equatorial boundary of the region of the substorm dipolarizations.

\section{Findings}

A relation between the minimum value of the Dst variation, maximum of partial ion pressure, and the location of the peak intensity of the relativistic electron fluxes

Analysis of the data of high apogee satellites during storms of different intensity showed that the location of the peak intensity of the relativistic electron fluxes appearing during the storm recovery phase $\left(L_{\max }\right.$ where $L_{\max }$ is the McIlwain parameter) depends on the amplitude of the Dst variation $\left(|D s t|_{\max }\right)$ according to the Tverskaya relation (Tverskaya
1986; Tverskaya et al. 2005): $|D s t|_{\max }=c\left(L_{\max }\right)^{-4}$ where $c$ is the coefficient of proportionality $c=2.75 \cdot 10^{4}$. The use of the SYM-H index instead of Dst gives the same relation, but the value of $c$ is slightly different, $c=3 \cdot 10^{4}$ (see the review of Tverskaya (2011)). The minimum Dst value for the 8-9 October 2012 magnetic storm is equal $-111 \mathrm{nT}$. This corresponds to $L_{\max }=3.97$. This value is in very good agreement with the results of Reeves et al. (2013). The Tverskaya dependence was verified for many magnetic storms and satellite observations (see Fig. 4). In this work, we add one more event to the curve: the 8-9 October 2012 geomagnetic storm (blue star). As it was shown in "The 8-9 October 2012 magnetic storm and the position of the auroral oval and of the westward auroral electrojet" section, the maximum of the ion density and the most equatorial boundary of the westward auroral electrojet were located at the same magnetic latitude in dipole approximation.

Tverskoy (1997) suggested an explanation why the Tverskaya relation has a power law dependence and obtained the value of power index by analyzing the magnetic field disturbance caused by the azimuthally symmetric plasma pressure distribution with the maximum at $L_{\max }$ and radial profile $L^{-7}$ for $L>L_{\max }$. Later, Antonova (2005, 2006) showed that it is even possible to obtain a theoretical explanation for the coefficient of proportionality in the Tverskaya relation. However, this theory was developed for the low $\beta$ plasma values (where $\beta$ is the plasma 


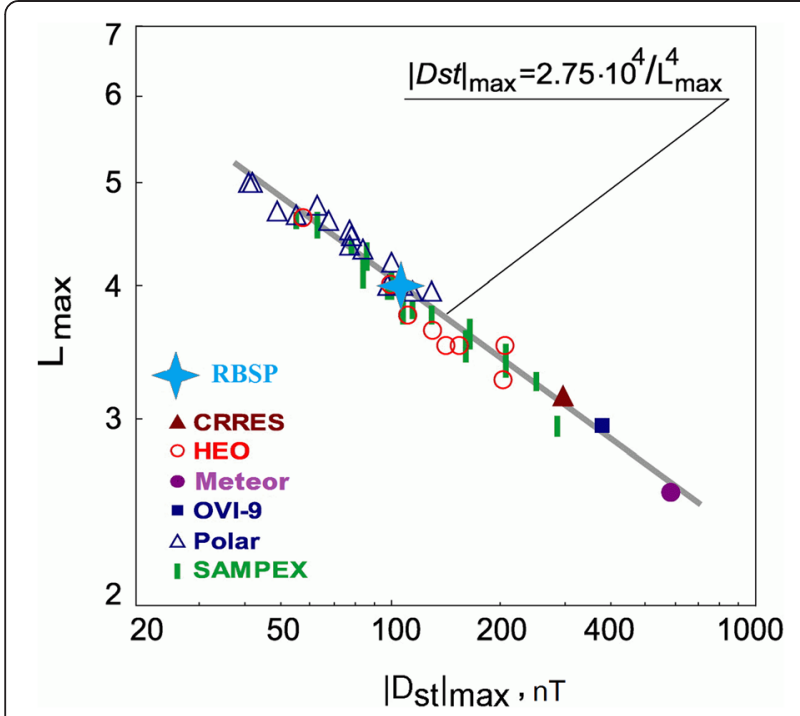

Fig. 4 Relation between the maximum variation of the $|D s t|$ and the location of the peak in the relativistic electron fluxes $\left(L_{\text {max }}\right)$. Experimental curve of the relation between $|D s t|$ and the peak intensity of the relativistic electron fluxes appearing during storm recovery phase. This diagram is adapted from Tverskaya's (2011) review, and a new event 8-9 October 2012 geomagnetic storm is added (blue star)

parameter) and predicts anomalously large increases in plasma pressure inside the magnetosphere. Vovchenko and Antonova (2012) showed that taking into account the nonlinear distortions of the dipole magnetic field by plasma for values of $\beta$ comparable with unity, it is possible to obtain flatter plasma pressure profile. However, the problem remains partially unsolved. The results of Tverskoy (1997) and Antonova $(2005,2006)$ show the importance of analyzing of the distribution of plasma pressure for determining of the mechanism of acceleration of the ORB electrons.

To clarify the radial dependence of plasma pressure, we calculated the low altitude radial ion plasma pressure using the data of the DMSP F16-18 satellites crossing the auroral oval between 19 and 24 magnetic local time (MLT). An example of such a crossing is given in Fig. 2. The plasma pressure was calculated using the data of ion precipitating fluxes measured by the SSJ/4 electrostatic analyzer within the energy range 0.03-30 keV. We used the methodology developed by Stepanova et al. $(2004,2006,2008)$ for quiet periods and for magnetic storms: this methodology considers the effects of the field-aligned potential drop on the ion precipitations. Mapping from the auroral altitudes into the equatorial plane was done using the IGRF and TS04 geomagnetic field models. Figure 5 shows the variation in ion plasma pressure with the distance from Earth, obtained using the IGRF (red circles) and TS04 (blue circles) models. As can be seen, the pressure maximum is

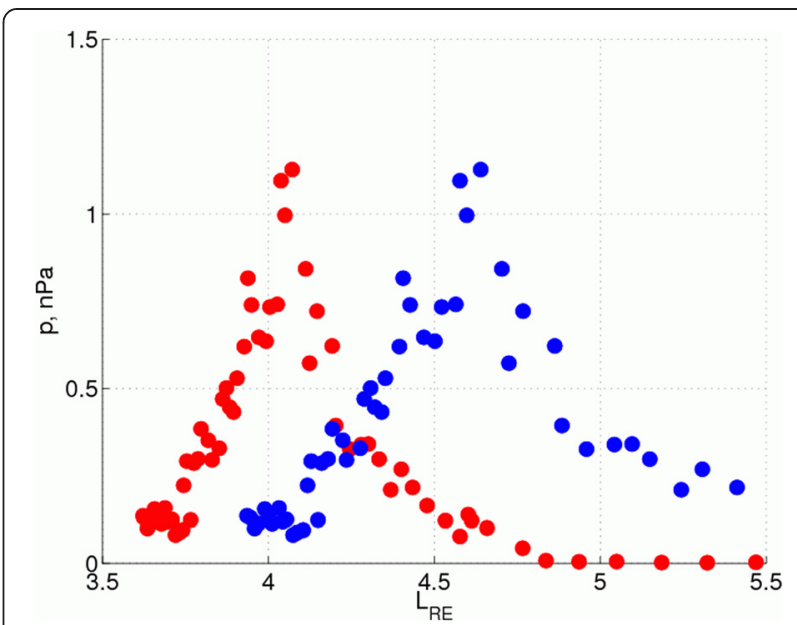

Fig. 5 Plasma pressure profiles obtained from the DMSP data shown in Fig. 2. Plasma pressure profiles obtained from the DMSP F17 satellite data during the auroral crossing on 8 October 2012 between 21:47:40 and 21:52:50 UT mapped to the equatorial plane by IGRF (red) and Tsyganenko 2004 (blue) models, we used the methodology proposed by Stepanova et al. $(2004,2006,2008)$

located at $R=4 R e$ in the case of the International Geomagnetic Reference Field (IGRF) mapping and at $R=4.5 R e$ in the case of the TS04 model. Analysis of other crossings near to the Dst minima showed that according to the IGRF model the pressure maxima are located between 3.5 and $4.2 R e$ and are located between 3.7 and 4.6 Re according to the TS04 model. The typical difference between the two models was around $0.4 R e$. As can be seen in Fig. 5, the plasma pressure profile is very sharp near the maximum; this result agrees with the predictions of Tverskoy (1997) and Antonova (2005, 2006).

As the DMSP SSJ/4 particle detectors analyzed the ion fluxes with the energies lower than $30 \mathrm{keV}$, the contribution of higher energies is estimated due to fits by Maxwellian or kappa functions. Nevertheless, the values of ion pressure shown in Fig. 5 could be underestimated considering possible deviations of distribution functions from Maxwellian or kappa and also considering the fact that the analyzer does not allow to determine the ion composition, which can be very important for the calculation of the full pressure (Krimigis et al. 1985). We also do not consider the electron contribution. The knowledge of the particle pressure for the full range of energies and species is important for the development of the theory of ORB acceleration taking into consideration that a diamagnetic decrease in the magnetic field $\sim 100 \mathrm{nT}$ in the region of maximum pressure can produce considerable betatron acceleration during ring current decay. However, we assume that the location of the maximum under the full particle pressure will not differ significantly from the location of the maximum under partial ion pressure. 
Reeves et al. (2013) concluded that chorus waves are responsible for the observed local acceleration of ORB electrons. However, Figure S4 in Reeves et al. (2013) shows high levels of chorus waves being measured by the EMFISIS instrument on board the RBSP-A satellite starting from the beginning of the storm till the noon of 9 October 2012 when the IMF was oriented northward. Nevertheless, the increase in particle fluxes was not observed till early morning of 9 October in spite of high level of chorus wave activity observed before. This means that the role of the chorus waves in the acceleration of relativistic electrons should be analyzed more carefully. Our analysis of the DMSP precipitating fluxes shows how the equatorial boundary of the auroral oval is moving toward the equator during the storm; this is in agreement with the classical picture of the auroral oval location. Auroral indexes shown in Fig. 1c also indicate strong substorm activity. During substorms, the wave activity is generally very high, especially when these substorms take place during storms. Therefore, conclusions about the dominant contribution of chorus waves in the acceleration of relativistic electrons need to be checked considering other possible mechanisms of electron acceleration. For example, it would be interesting to understand the contribution of the interaction of electrons with nonlinear waves, in accordance with results of Omura and Summers (2006) and Demekhov et al. (2006). It is also necessary to analyze the form of the spectra of the seed population of electrons and to exclude the contribution of simple betatron acceleration of the seed electrons injected in the region of depressed magnetic field during the storm recovery phase, which could be significant.

\section{Conclusions}

In this study, we analyze the auroral and electrojet dynamics during a moderate geomagnetic storm. We use the data of auroral particle precipitating fluxes measured by the DMSP satellites and the data of the location of the westward electrojet from the IMAGE magnetometer network obtained during the 8-9 October 2012 magnetic storm. We show that the maximum of the relativistic electron fluxes filling the ORB is located at the same $L$ as the maximum in the ion pressure obtained in the energy range till $30 \mathrm{keV}$ at low latitudes. This maximum coincides with the position the maximal shift of the westward electrojet toward the equator. We argue that the high level of substorm activity, observed during this storm, could create a seed population of electrons in the region of maximum plasma pressure at comparatively low latitudes (for the analyzed storm, near the $60^{\circ} \mathrm{MLAT}$ ), which could be accelerated by a betatron mechanism during the recovery phase. However, this hypothesis requires additional analysis of the possible contribution of adiabatic acceleration during ring current decay.

\section{Abbreviation}

ORB: outer electron radiation belt.

\section{Competing interests}

The authors declare that they have no competing interests.

\section{Authors' contributions}

All authors have contributed equally. Both authors read and approved the final manuscript.

\section{Authors' information}

The authors have been involved in a collaboration with Moscow State University and Universidad de Santiago de Chile for many years.

\section{Acknowledgements}

We acknowledge Oleg Troshichev, Alexander Janzhura, and Jürgen Matzka for the data of the Polar Cap (PC) index. The OMNI data were obtained from the GSFC/SPDF OMNIWeb interface at http://omniweb.gsfc.nasa.gov. Detectors were designed by Dave Hardy of AFRL and data obtained from JHU/APL. This work was supported by the Dirección de Investigación Científica y Tecnológica (DICYT), Vicerrectoria de Inverstigación Desarrollo e Innovación (VRIDEI), Universidad de Santiago de Chile (M. Stepanova), grants: DICYT 041531SSSA, 041531SSSA_INTEXCELE and 041531SSSA_APOYOQ1, and by the grant of Russian Foundation for Basic Research No 15-05-04965 (E.E. Antonova).

\section{Author details}

${ }^{1}$ Skobeltsyn Institute of Nuclear Physics, Lomonosov Moscow State University, Moscow, Russia. ${ }^{2}$ Physics Department, Science Faculty, Universidad de Santiago de Chile (USACH), Santiago, Chile.

Received: 1 May 2015 Accepted: 28 August 2015

Published online: 15 September 2015

\section{References}

Akasofu SI, Chapman S (1963) The lower limit of latitude (US sector) of northern quite auroral arcs, and its relation to Dst(H). J Atmos Terr Phys 25:9-12. doi:10.1016/0021-9169(63)90011-4

Antonova EE (2005) Magnetospheric substorms and the sources of inner magnetosphere particle acceleration. In: Pulkinen TI, Tsyganenko NA, Friedel RHW (eds) The inner magnetosphere: physics and modeling, geophysical monograph series vol 155. AGU, Washington, pp 105-111

Antonova EE (2006) Stability of the magnetospheric plasma pressure distribution and magnetospheric storms. Adv Space Res 38:1626-1630. doi:10.1016/ j.asr.2005.05.005

Antonova EE, Kirpichev IP, Vovchenko W, Stepanova MV, Riazantseva MO, Pulinets MS, Ovchinnikov IL, Znatkova SS (2013) Characteristics of plasma ring, surrounding the Earth at geocentric distances $\sim 7-10 R_{E}$, and magnetospheric current systems. J Atmos Sol Terr Phys 99:85-91. doi:10.1016/j.jastp.2012.08.013

Antonova EE, Kirpichev IP, Stepanova MV (2014a) Plasma pressure distribution in the surrounding the Earth plasma ring and its role in the magnetospheric dynamics. J Atmos Sol Terr Phys 115-116:32-40. doi:10.1016/j.jastp.2013.12.005

Antonova EE, Vorobjev VG, Kirpichev IP, Yagodkina OI (2014b) Comparison of the plasma pressure distributions over the equatorial plane and at low altitudes under magnetically quiet conditions. Geomagn Aeron 54:278-281. doi:10.1134/S0016793214030025

Baker DN, Elkington SR, Li X, Wiltberger MJ (2005) Particle acceleration in the inner magnetosphere. In: Pulkinen TI, Tsyganenko NA, Friedel RHW (eds) The inner magnetosphere: physics and modeling, geophysical monograph series vol 155. AGU, Washington, pp 73-85

Burton RK, McPherron RL, Russell CT (1975) An empirical relationship between interplanetary conditions and Dst. J Geophys Res 80:4204-4214. doi:10.1029/ JA080i031p04204

Demekhov AG, Trakhtengerts VY, Rycroft MJ, Nunn D (2006) Electron acceleration in the magnetosphere by whistler-mode waves of varying frequency. Geomagn Aeron 46:711-716. doi:10.1134/S0016793206060053

King JH, Papitashvili NE (2005) Solar wind spatial scales in comparisons of hourly wind and ACE plasma and magnetic field data. J Geophys Res. doi:10.1029/2004JA010649

Kissinger J, Kepko L, Baker DN, Kanekal S, Li W, McPherron RL, Angelopoulos V (2014) The importance of storm time steady magnetospheric convection in 
determining the final relativistic electron flux level. J Geophys Res 119:7433-7443. doi:10.1002/2014JA019948

Krimigis SM, Gloeckler G, McEntire RW, Potemra TA, Scarf FL, Shelley EG (1985) Magnetic storm of September 4, 1984: a synthesis of ring current spectra and energy densities measured with AMPTE/CCE. Geophys Res Lett 12:329-332. doi:10.1029/GL012i005p00329

Newell PT, Feldstein YI, Galperin Yl, Meng C-I (1996) Morphology of nightside precipitation. J Geophys Res 101:10737-10748. doi:10.1029/95JA03516

Newell PT, Liou K, Wilson GR (2009) Polar cap particle precipitation and aurora: review and commentary. J Atmos Sol Terr Phys 71:199-215. doi:10.1016/ j.jastp.2008.11.004

Omura Y, Summers D (2006) Dynamics of high-energy electrons interacting with whistler mode chorus emissions in the magnetosphere. J Geophys Res. doi:10.1029/2006JA011600

Reeves GD (1998) Relativistic electrons and magnetic storms: 1992-1995. Geophys Res Lett 25:1817-1820. doi:10.1029/98GL01398

Reeves GD, McAdams KL, Friedel RHW, O'Brien TP (2003) Acceleration and loss of relativistic electrons during geomagnetic storms. Geophys Res Lett. doi:10.1029/2002GL016513

Reeves GD, Spence E, Henderson MG, Morley SK, Friedel RHW, Funsten HO, Baker DN, Kanekal SG, Blake JB, Fennell JF, Claudepierre SG, Thorne RM, Turner DL, Kletzing CA, Kurth WS, Larsen BA, Niehof JT (2013) Electron acceleration in the heart of the Van Allen radiation belts. Science 341:991. doi:10.1126/science.1237743

Stepanova MV, Antonova EE, Bosqued JM, Kovrazhkin R (2004) Azimuthal plasma pressure reconstructed by using the Aureol-3 satellite data during quiet geomagnetic conditions. Adv Space Res 33:737-741. doi:10.1016/S0273-1177(03)00641-0

Stepanova MV, Antonova EE, Bosqued JM (2006) Study of plasma pressure distribution in the inner magnetosphere using low-altitude satellites and its importance for the large-scale magnetospheric dynamics. Adv Space Res 38:1631-1636. doi:10.1016/j.asr.2006.05.013

Stepanova MV, Antonova EE, Bosqued JM (2008) Radial distribution of the inner magnetosphere plasma pressure using low-altitude satellite data during geomagnetic storm: the March 1-8, 1982 event. Adv Space Res 41:1658-1665. doi:10.1016/j.asr.2007.06.002

Tsyganenko NA, Sitnov MI (2005) Modeling the dynamics of the inner magnetosphere during strong geomagnetic storms. J Geophys Res. doi:10.1029/2004JA010798

Tverskaya LV (1986) On the boundary of electron injection into the magnetosphere. Geomagn Aeron 26:864-865

Tverskaya LV (2011) Diagnostics of the magnetosphere based on the outer belt relativistic electrons and penetration of solar protons: a review. Geomagn Aeron 51:6-22. doi:10.1134/S0016793211010142

Tverskaya LV, Ivanova TA, Pavlov NN, Ya Reizman S, Rubinstein IA, Sosnovets EN, Veden'kin NN (2005) Storm-time formation of a relativistic electron belt and some relevant phenomena in other magnetosphere plasma domains. Adv Space Res 36:2392-2400. doi:10.1016/j.asr.2003.09.071

Tverskoy BA (1997) Formation mechanism for the structure of the magnetic storm ring current. Geomagn Aeron 37:555-559 (in Russian)

Vovchenko W, Antonova EE (2012) Dependence of volumes of magnetic flux tubes on plasma pressure and disturbance in the magnetic field in the axially symmetric case. Geomagn Aeron 52:49-59. doi:10.1134/S0016793212010161

\section{Submit your manuscript to a SpringerOpen ${ }^{\circ}$ journal and benefit from:}

- Convenient online submission

- Rigorous peer review

- Immediate publication on acceptance

- Open access: articles freely available online

- High visibility within the field

- Retaining the copyright to your article

Submit your next manuscript at $>$ springeropen.com 